\title{
Extrinsic Elements Extraction of DGMESFET
}

\author{
Wei-Kung Deng and Tah-Hsiung Chu \\ Department of Electrical Engineering, \\ National Taiwan University, Taipei, Taiwan 10617, ROC
}

\begin{abstract}
A procedure for the extraction of extrinsic elements of dual-gate MESFET (DGMESFET) is described in this paper. It is the first time to accurately extract the extrinsic elements of series resistance by considering the distributed channel resistance under the regions of two gates with the use of "end resistance measurement" method. The extrinsic elements of capacitance and inductance are extracted by three-port Y-matrix and Z-matrix calculation from cold measurements. The developed procedure is useful for the characterization of DGMESFET.
\end{abstract}

\section{Introduction}

To design a microwave circuit using DGMESFET, an equivalent circuit at operational bias condition is required. The small-signal models [1]-[3] and large-signal model [4] have been proposed by many authors. In those papers, the equivalent circuit of DGMESFET is basically composed by cascoding two single gate MESFETs (SGMESFET) embedded by extrinsic parasitic elements. Fig. 1 shows a typical small-signal equivalent circuit of a coplanar DGMESFET. Since the connection between two intrinsic FETs can not be directly measured from probes, it is not easy to find the analytical formula to extract the intrinsic and extrinsic elements as in the SGMESFET case [5]. In this paper, we will describe the developed extraction procedure for the extrinsic elements of DGMESFET.

For a DGMESFET, the values of extrinsic series resistance can be estimated from physical modeling [1],[6] or derived by empirical formula with the distributed channel resistance under the regions of two

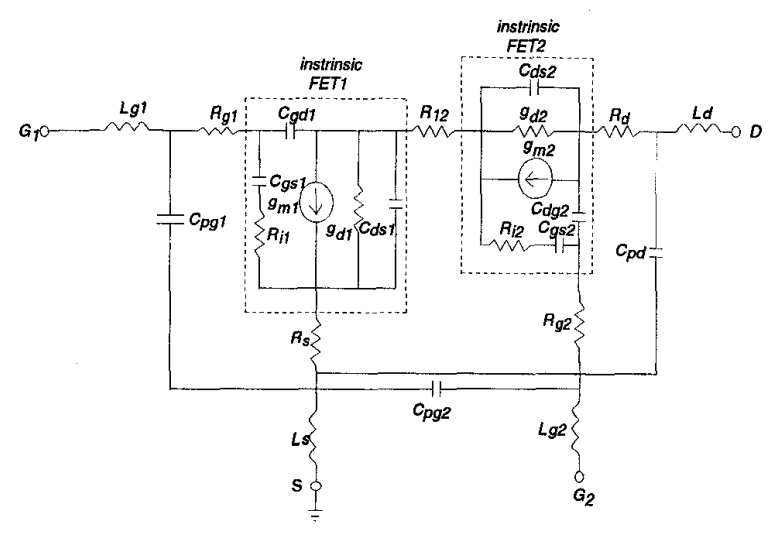

Fig.1 A small-signal equivalent circuit of DGMESFET.

gates to be neglected [2],[3]. In this paper, a circuit model of cold DGMESFET is proposed to consider the distributed channel resistance. The precise values of extrinsic series resistance can then be extracted by using the "end resistance measurement" method [7]. The extrinsic elements of capacitance and inductance are extracted using three-port $Y$-parameter and Z-parameter calculation from the cold measurements with DGMESFET at forward bias and reverse bias accordingly.

\section{Series Extrinsic Resistance Extraction}

The schematic diagram to describe the DC behavior of a cold DGMESFET under forward bias is shown in Fig.2. Five independent equations with seven unknowns can be acquired from the "end resistance measurement" method as follows

$$
\left.\frac{\Delta V_{g 1 s}}{\triangle I_{g 1 s}}\right|_{D \text { rainfloating }}=R_{g 1}+\frac{n_{1} V_{T}}{I_{g 1 s}}+\frac{R_{c 1}}{3}+R_{s},
$$




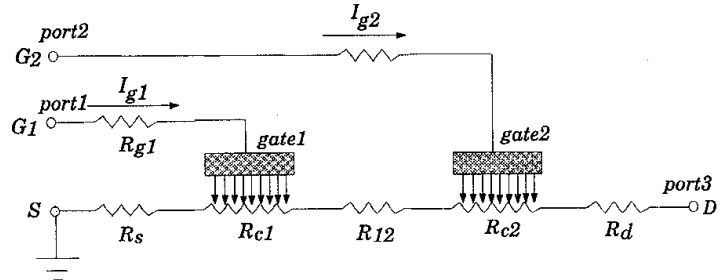

Fig. 2 Schematic diagram of a DGMESFET for "end resistance measurement" method. $R_{g 1}, R_{g 2}, R_{s}$ and $R_{d}$ are gate1, gate2, source, and drain resistances. $R_{c 1}, R_{c 2}$ are the distributed channel resitance under the regions of gatel and gate, respectively. $R_{12}$ is the bulk resitance between gate1 and gate2.

$$
\begin{gathered}
\left.\frac{\Delta V_{g 2 s}}{\Delta I_{g 2 s}}\right|_{\text {Drainfloating }}=R_{g 2}+\frac{n_{2} V_{T}}{I_{g 2 s}}+\frac{R_{c 2}}{3}+R_{12} \\
+R_{c 1}+R_{d} \\
\left.\frac{\Delta V_{g 2 d}}{\Delta I_{g 2 d}}\right|_{\text {Sourcefloating }}=R_{g 2}+\frac{n_{2} V_{T}}{I_{g 2 d}}+\frac{R_{c 2}}{3}+R_{d} \\
\left.\frac{\Delta V_{g 1 d}}{\triangle I_{g 1 d}}\right|_{\text {Sourcefloating }}=R_{g 1}+\frac{n_{1} V_{T}}{I_{g 1 d}}+\frac{R_{c 1}}{3}+R_{12} \\
+R_{c 2}+R_{d} \\
\left.\frac{V_{d s}}{I_{g 1 s}}\right|_{\text {Drainfloating }}=R_{c 1}+R_{s}
\end{gathered}
$$

Equation 1 shows a straight line for $V_{g 1 s}-I_{g 1 s}$ characteristics with $n_{1} V_{T}$ as the slope and $R_{g 1}+\frac{R_{c 1}}{3}+$ $R_{s}$ as the intercept point. Therefore from a set of measurements of current $I_{g 1 s}$ and voltage $V_{g 1 s}$ with floating drain terminal, the mean values of $\frac{\Delta V_{g 1 s}}{\Delta I_{g 1 s}}$ can be calculated to solve $n_{1} V_{T}$ and $R_{g 1}+\frac{R_{c} 1}{3}+R_{s}$ by a least square error method. By using the same process, (1)-(4) become the following three-port Z-parameter expression

$$
\begin{gathered}
R_{11}=R_{g 1}+\frac{R_{c 1}}{3}+R_{g} \\
R_{22}=R_{g 2}+\frac{R_{c 2}}{3}+R_{12}+R_{c 1}+R_{d} \\
R_{32}=R_{g 2}+\frac{R_{c 2}}{3}+R_{d} \\
R_{31}=R_{g 1}+\frac{R_{c 1}}{3}+R_{12}+R_{c 2}+R_{d}
\end{gathered}
$$

and (5) can be rewritten as

$$
R_{x}=\frac{R_{c 1}}{2}+R_{s}
$$

Two additional equations for solving seven extrinsic elements of series resistance can be selected from the following approaches

1 the relation of $R_{c 1}$ and $R_{c 2}, R_{c 1}=m R_{c 2}$ provided the gate1 and gate 2 channel length ratio $m$ is known.

2 the value of $R_{s}+R_{12}+R_{c 2}+R_{d}$ obtained from Hower and Bechte method [8] by floating gate 2 terminal.

3 the values of $R_{g 1}$ and $R_{g 2}$ acquired from dummy pad resistance measurement.

4 the values of $R_{s}$ and $R_{d}$ of SGMESFET [5] provided the same device structure and processing in DGMESFET for source and drain terminals.

\section{Extrinsic Capacitance Extraction}

Figure 3 shows the equivalent circuit of a cold coplanar DGMESFET in which FET1 is reverse biased in pinched-off region and FET2 is forward biased in linear region. The imaginary part of the three-port $\mathrm{Y}$ parameter, with frequency below a few gigaherhz, can be written as

$$
\begin{gathered}
\operatorname{Im}\left(Y_{11}\right)=j \omega\left(C_{p g 1}+2 C_{b 1}\right), \\
\operatorname{Im}\left(Y_{13}\right)=-j \omega(C b 1), \\
\operatorname{Im}\left(Y_{22}\right)=j \omega\left(2 C_{g 2 d}+2 C_{p g 2}\right), \\
\operatorname{Im}\left(Y_{23}\right)=-j \omega\left(2 C_{g 2 d}\right), \\
\operatorname{Im}\left(Y_{33}\right)=j \omega\left(C_{p d}+2 C_{g 2 d}+C b 1\right) .
\end{gathered}
$$

One can then solve the values of extrinsic capacitance $C_{p g 1}, C_{p g 2}, C_{p d}$ and $C_{g 2 d}$.

\section{Extrinsic Inductance Extraction}

Figure 4 shows the equivalent circuit of a coplanar cold DGMESFET with FET1 and FET2 both at forward bias. The real part of the three-port Zparameter, with frequency below a few gigahertz, can be written as

$$
\begin{aligned}
& \operatorname{Re}\left(Z_{11}\right)=j \omega\left(L_{g 1}+L_{s}\right) \\
& \operatorname{Re}\left(Z_{22}\right)=j \omega\left(L_{g 2}+L_{s}\right)
\end{aligned}
$$




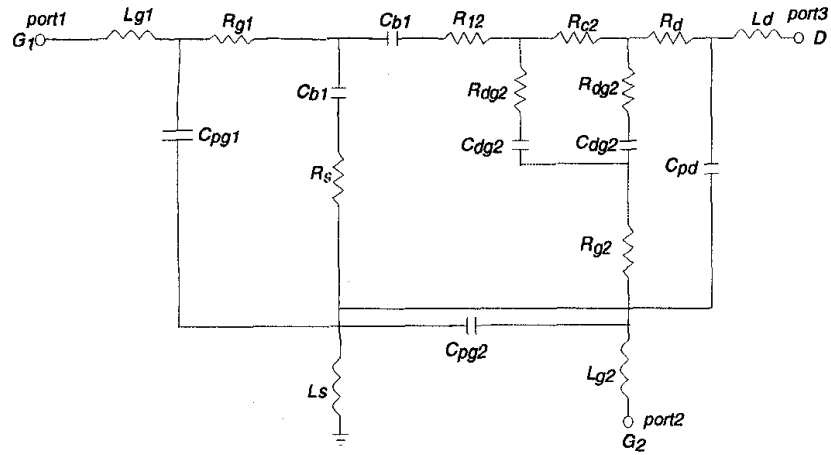

Fig. 3 An equivalent circuit of coplanar cold DGMESFET with FET1 reverse biased and FET2 forward biased.

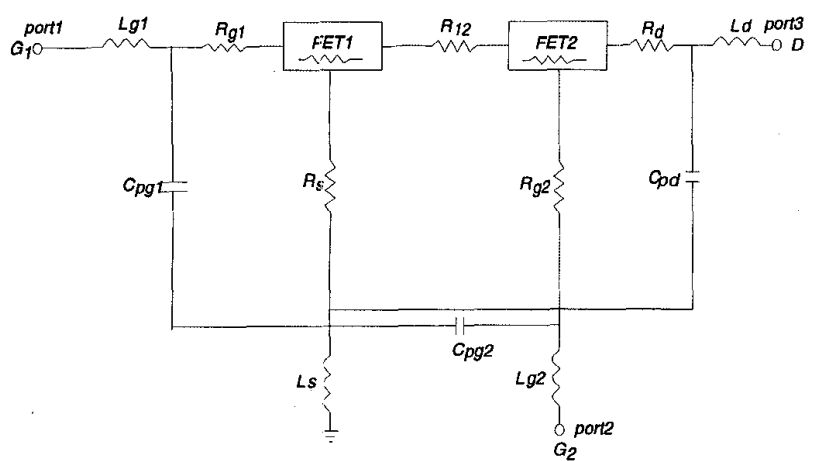

Fig. 4 An equivalent circuit of coplanar cold DGMESFET with FET1 and FET2 forward biased.

$$
\begin{gathered}
\operatorname{Re}\left(Z_{33}\right)=j \omega\left(L_{d}+L_{s}\right), \\
\operatorname{Re}\left(Z_{21}\right)=j \omega L_{s} .
\end{gathered}
$$

One can then solve the values of extrinsic inductance $L_{g 1}, L_{g 2}, L_{s}$ and $L_{d}$.

\section{Measurement Results}

A coplanar DGMESFET with $L_{g 1}, L_{g 2}=1 \mu \mathrm{m}$ and $W_{g 1}, W_{g 2}=4 \times 75 \mu m$ fabricated by HEXAWAVE Co., is measured to extract the extrinsic elements. The three-port scattering matrix of DGMESFET is measured on wafer using an automated three-port network analyzer with associated calibration method developed in our laboratory. Figure 5 shows the measured results of $\frac{\Delta V_{g 1 s}}{\Delta I_{g 1 s}}, \frac{\Delta \dot{V}_{g 1 d}}{\Delta I_{g 1 d}}, \frac{\Delta V_{g 2 s}}{\Delta I_{g 2 s}}$ and $\frac{\Delta V_{g 2 d}}{\Delta I_{g 2 d}}$ with floating drain terminal or source terminal, respectively. The three-port Y-parameter at biases $V_{g 1 s}<V_{t h}, I_{g 2}=$

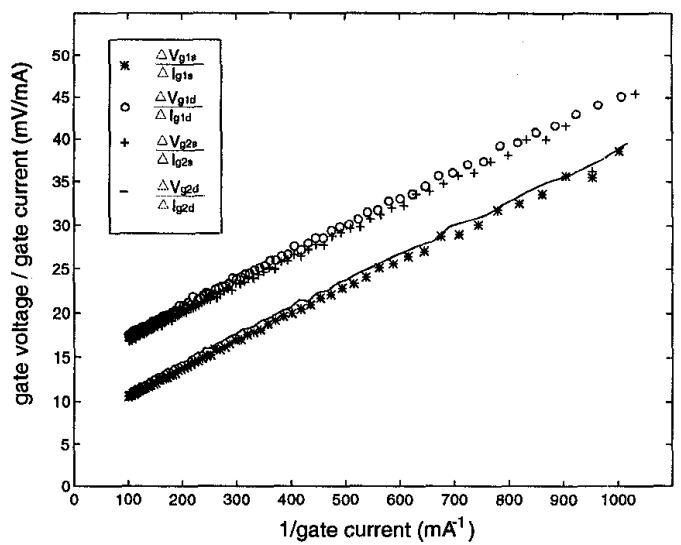

Fig. 5 Measured results of $\frac{\Delta V_{g 1 s}}{\Delta I_{g 1 s}}, \frac{\Delta V_{g 1 d}}{\Delta I_{g 1 d}}, \frac{\Delta V_{g 2 s}}{\Delta I_{g 2 s}}$, and $\frac{\Delta V_{g 2 d}}{\Delta I_{g 2 d}}$ at forward bias with floating drain terminal or source terminal, respectively.

$0.2 m A$ and $V_{d s}=0 \mathrm{~V}$ are calculated from the onwafer measurement of S-parameter. The results of $\operatorname{Im}\left(Y_{11}\right), \operatorname{Im}\left(Y_{22}\right), \operatorname{Im}\left(Y_{21}\right)$ and $\operatorname{Im}\left(Y_{33}\right)$ are shown in Fig. 6. Values of $C_{p g 1}, C_{p g 2}, C_{p d}$ and $C_{b}$ calculated from (11)-(15) are shown in Fig.7, and values of extrinsic inductance $L_{s}$ and $L_{d}$ are shown in Fig. 8. Table 1 summaries the extraction results of extrinsic elements of DGMESFET and the associated bias conditions.

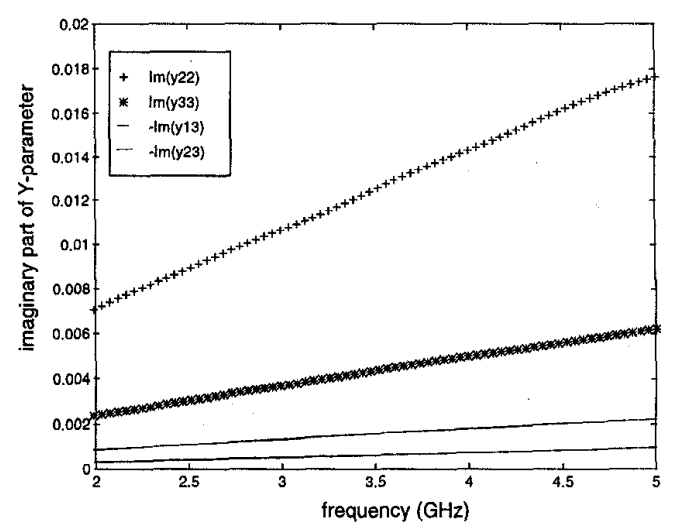

Fig. 6 Measured results of the imaginary part of three-ort $Y$ parameter, $V_{d s}=0 V, V_{g 1 s}=V_{t h}$, and $I_{g 2 s}=0.2 \mathrm{~mA}$.

\section{References}

[1] S. Asai, F. Murai, and H. Kodera, "GaAs dualgate schottky-barrier FET's for frequencies," 


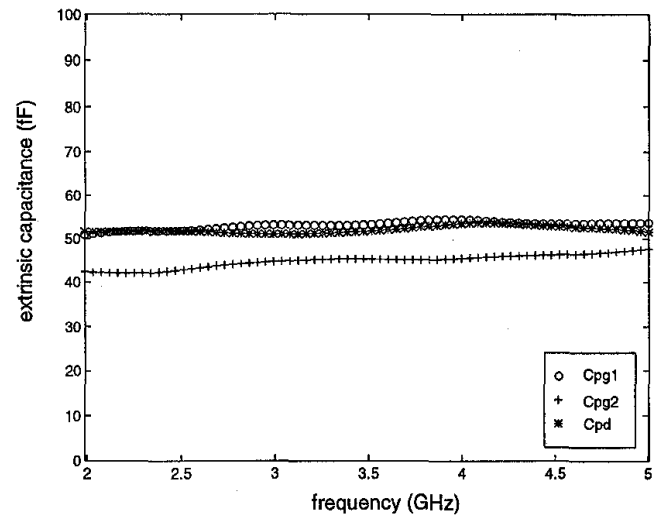

Fig. 7 Measured results of extrinsic elements of capacitance.

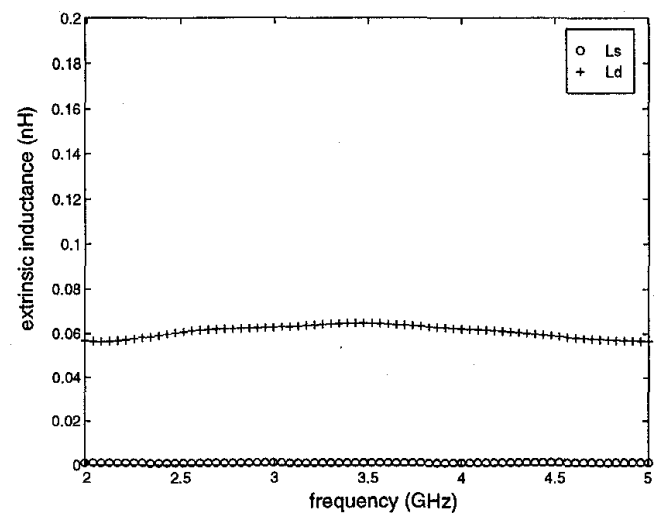

Fig. 8 Measured results of extrinsic elements of inductance.

IEEE Trans. Electron Devices, vol. ED-22, pp. 897-904, Oct. 1975.

[2] C. Tsironis and R. Meierer, "Microwave wideband model of GaAs dual-gate MESFET," IEEE Trans. Microwave Theory and Tech,, vol. MTT30, PP. 243-251, Mar. 1982.

[3] M. Schöön, "A novel, bias-dependent, smallsignal model of the dual-gate MESFET," IEEE Trans. Microwave Theory and Tech., vol. MTT42, July 1988.

[4] A. Madjor and J. Dreifuss, "Large-signal microwave performance prediction of dual-gate MESFET using an efficient and accurate model," IEEE Trans. Microwave Theory and Tech., vol. MTT-33, pp. 693-643, July 1985.

[5] G. Dambrine, A. Cappy, F. Heliodore, and E.

\begin{tabular}{|c|c|c|}
\hline \multicolumn{3}{|c|}{ cold measurement $(\mathrm{Vds}=0 \mathrm{~V})$} \\
\hline Extrinsic Resistance (ohm) & Extrinsic Capacitance (fF) & Extrinsic Inductance $(\mathrm{nH})$ \\
\hline $\begin{array}{r}\text { FET1: forward bjas } \\
\text { / gl=1-10mA }\end{array}$ & $\begin{array}{c}\text { FET1: reverse blas } \\
\vee \text { g1s< Vth }\end{array}$ & $\begin{array}{r}F E T 1: \text { forward bias } \\
\text { I gt }=0.2 \mathrm{~mA}\end{array}$ \\
\hline $\begin{array}{r}\text { FET2: forward bias } \\
\text { / gl=1-10mA } \\
\text { with floating drain or source }\end{array}$ & $\begin{array}{c}\text { FET2: forward bias } \\
\qquad \mathrm{gt}=0.2 \mathrm{~mA}\end{array}$ & $\begin{array}{c}\text { FET2: forward bias } \\
\text { / g } 1=0.2 \mathrm{~mA}\end{array}$ \\
\hline $\begin{array}{l}R g 1=1.59 \\
R g 2=1.52 \\
R s=3.68 \\
R d=3.59 \\
R 12=3.1 \\
R c 1=3.05 \\
R c 2=3.05\end{array}$ & $\begin{array}{l}\text { Cpg } 1=53 \\
\text { cpg2 }=44 \\
C p d=52\end{array}$ & $\begin{array}{l}L s=0.003 \\
L d=0.066 \\
L g 1=0.002 \\
L g 2=0.002\end{array}$ \\
\hline
\end{tabular}

Table 1 Values of extrinsic elements of DGMESFET and measured bias condition.

Playez, "A new method for determining the FET small-signal equivalent-circuit," IEEE Trans. Microwave Theory and Tech, vol. MTT-36, pp. 1151-1159, July 1988.

[6] T. Furutsuka, M. Ogawa, and N. Kawamura, "GaAs dual-gate MESFET's," IEEE Trans. Electron Devices, vol. ED-25, pp. 580-586, June 1978.

[7] K. W. Lee et al, "Source, drain, and gate series resistances and electron saturation velocity in ionimplanted GaAs FET's," IEEE Trans. Electron Devices, vol. ED-32, pp. 982-992, May 1985.

[8] P. L. Hower and N. G. Bechtel, "Current saturation and small-signal characteristics of GaAs FET's," IEEE Trans. Electron Devices, vol. ED20, Mar. 1973. 